\title{
Efficacy of a Program of Prevention and Control for Methicillin-Resistant Staphylococcus aureus Infections in an Intensive-Care Unit
}

\author{
Marina Moreira ${ }^{1}$, Marise R. Freitas ${ }^{2}$, Sinaida T. Martins ${ }^{3}$, Adauto Castelo ${ }^{3}$ and Eduardo Alexandrino Servolo Medeiros $^{3}$ \\ ${ }^{1}$ Taubaté University, Taubaté, SP; ${ }^{2}$ Federal University of Rio Grande do Norte, Natal, RN; ${ }^{3}$ Federal University of São Paulo, São Paulo, SP, Brazil
}

\begin{abstract}
Methicillin-resistant Staphylococcus aureus (MRSA) is endemic in most Brazilian Hospitals, and there are few studies which show the efficacy of control measures in such situations. This study evaluated intensive care unit (ICU) patients, in two years divided in control, intervention and post-intervention group. Intervention measures: hands-on educational programs for healthcare workers; early identification of MRSA infected or colonized patients, labeled with a bed-identification tag for contact isolation; nasal carriers, patients, and healthcare professionals treated with topical mupirocin for five days. The hospital infection rates in the control period were compared to the ones in the post-intervention period. Hospital infection rates were found by means of the NNISS methodology The incidence coefficients of MRSA hospital infection (monthly average of $1,000 \mathrm{pts} / \mathrm{day}$ ) in the control, intervention and post-intervention groups were respectively: 10.2, 5.1 and $2.5 / 1,000 \mathrm{pts} /$ day $(p<0.001)$ and MRSA-originated bloodstream infections were $3.6,0.9$ and $1.8 / 1,000$ central venous catheter/day $(p=0.281)$. Nasal colonization in both intervention and post-intervention periods was of $30.9 \%$ and $22.1 \%$ among the hospitalized patients, respectively $54.4 \%$ and $46.1 \%$ of whom were already MRSA-positive when admitted to the unit. In the intervention period, most of those MRSA infected patients (76.2\%) were nasal carrier. Mortality rates were, respectively $26.6 \% ; 27.3 \%$ and $21.0 \%(p<0.001)$. Nasal carriers, both patients $(93.7 \%)$ and healthcare professionals $(88.2 \%)$, were successfully treated with topical mupirocin. Intervention measures for the prevention and control of MRSA infections in ICUs, have been efficient in the reduction of the bloodstream and MRSA-originated hospital infections incidence, and reduced the overall mortality rate significantly.
\end{abstract}

Key-Words: MRSA, methicillin-resistant Staphylococcus aureus, intensive care unit infections.

Methicillin-resistant Staphylococcus aureus (MRSA) is currently deemed a ubiquitous pathogen, that gives rise to community acquired infections, endemic and epidemic hospital infection and colonization. Concern about infections arising from such microorganism increases as changes in its epidemiology occur, with an increased number of community acquired cases ${ }^{1}$ and the emergence of vancomycin-reduced susceptibility strains [1,2]. High prevalence of MRSA in the large Brazilian public and university hospitals has been found. In our setting, $73.2 \%$ of bloodstream $S$. aureus hospital infections arose from MRSA [3]. ICUs are important reservoirs in the MRSA epidemiology, and they may be a source of dissemination to other areas of the hospital [3-5].

Studies on MRSA control measures are controversial, on account of the diversity of situations which require intervention [6]. Some authors believe that no effort to control the microorganism should be made unless upon outbreaks or to prevent the emergence of antibiotics resistance $[4,7,8]$. Evaluated single measures or measures in different combinations proved to be effective even at MRSA endemic level institutions [5,7,9].

There are evidence that MRSA nasal carriers contribute towards the development of infections by such agent, in critically ill patientes [10], such evidence including decreased

Received on 14 July 2006; revised 8 January 2007.

Address for correspondence: Dr. Marina Moreira. Rua Peru, $\mathrm{n}^{\circ} 175$ Bairro: Jardim das Nações. Taubaté, SP. Zip code: 12030-280. E-mail: Marinamor@itelefonica.com.br. Phone: (12) 3632-8781/(12) 97822274.

The Brazilian Journal of Infectious Diseases 2007;11(1):57-62. (C) 2007 by The Brazilian Journal of Infectious Diseases and Contexto Publishing. All rights reserved. infection rates by the microorganism after treatment of narecolonized patients [5,9,11]. Mupirocin topic antibiotic of excellent antibacterial activity against $S$. aureus, including MRSA, is currently deemed the drug of choice for decolonization [12,13], although its long-term use may give rise to resistant strains. [14]

\section{Materials and Methods}

Setting

Hospital São Paulo (São Paulo, Brazil) - São Paulo Federal University tertiary-care referral university hospital, with 600 beds and 10 intensive-care units. The unit studied is a general ICU for adults with 16 beds, 10 of which are distributed throughout a large ward and six throughout smaller individual wards.

\section{Patients and Methods}

Prospective cohort study conducted on all patients hospitalized consecutively in the unit, who met the hospital infections epidemiologic surveillance enrollment criteria according to the National Nosocomial Infections Surveillance System (NNISS) [15], from June 1, 1996 to June 30, 1998 and divided, by periods, into control, intervention and postintervention groups.

\section{Control Group}

June 1, 1996 through October 31, 1996, period wrich the unit routine was kept, in compliance with the NNISS hospital infections surveillance, intensive-care unit component.

Intervention Group

November 1, 1996 through October 31, 1997. Besides the 
NNISS hospital infection surveillance, culture from the anterior nares of all of the unit inpatients was collected, within the first 48 hours of hospitalization and, after that, on a weekly basis until discharge or death. The healthcare workers (HCWs) also had culture from their anterior nares collected as of March 1997, which was repeated every two months.

Anterior nare samples were collected by using an auginate swab (Bionet ${ }^{\circledR}$ ) and taken to the Clinical Microbiology Special Laboratory in $0.7 \%$ semi-solid nutrient agar (Difco ${ }^{\circledR}$ ). Each sample was cultured on a blood agar plate supplemented with $5 \%$ defibernized sheep blood and inoculated in triptona soy broth vials (TSB-Oxoid ${ }^{\circledR}$ ), followed by a 24 -hour $35^{\circ}-37^{\circ} \mathrm{C}$ aerobic inoculation. Plate-isolated colonies suspected of staphylococci contamination (morphological characteristics, presence of hemolysis, and, pigment production analyzed) were presumptively identified by Gram staining, followed by another 24 hour isolation in blood agar, before they were submitted to the Staph-test for serologic evidence (Difco ${ }^{\circledR}$ ).

The samples were assessed for oxacillin and mupirocin susceptibility, by means of Disk Diffusion susceptibility tests and agar screening (Gold standard), in compliance with the procedures set out by the NCCLS (1997a, 1997b) [16,17]. For every sample, a bacterial growth suspension was prepared in 5 mL of Mueller-Hinton broth (MHB-Oxoid ${ }^{\circledR}$ ) and turbidity was adjusted to 0.5 on the McFarland scale by means of an Abbott-A-Just (Abbott ${ }^{\circledR}$ ) nephelometer. Following homogenization of the suspension, the suspension was cultured on a plate containing Mueller-Hinton Agar (MHAOxoid $\left.^{\circledR}\right)$ and later antibiotic disks were added: oxacillin (1 $\mu \mathrm{g}$ Sensifar $^{\circledR}$ ) and mupirocin (5 $\mu$ g-Sensifar $\left.{ }^{\circledR}\right)$. The plates were incubated at $35^{\circ} \mathrm{C}$ for $18-24$ hours, and their halo was then measured. Oxacillin-resistance was considered a halo diameter growth inhibition equal to or smaller than $10 \mathrm{mM}$, or one smaller than or equal to $14 \mathrm{mM}$ for mupirocin. The isolate was swabplated on Mueller-Hinton Agar supplemented with $4 \% \mathrm{NaCl}$ and oxacillin $(6 \mu \mathrm{g} / \mathrm{mL})$. The plates were incubated at $35^{\circ} \mathrm{C}$ for 24 hours for later reading. For the control of both tests, strains of Staphylococcus aureus ATCC 25923-oxacillin-sensitive and Staphylococcus aureus ATCC 33591- oxacillin-resistant, were used.

Cultures from other sites, such as tracheal secretions, skin and urine, were collected according to service routine, which was not changed during the study. From April 1997 on, along with the nasal swab collection, HCWs had an educational measures program implemented. Such guidance was provided in the unit itself through explanatory classes to nurse aids and nurse technicians, hygiene and administrative professionals, within their working hours, focusing on the epidemiology of the microorganism particularly on its transmission mechanisms and control measures. During the classes, the importance of handwash was emphasized, as well as the need for each HCW to take on responsibility for the control of infections. A lecture was given to physicians, nurses and respiratory therapists, emphasizing the need to implement control measures to prevent MRSA spread, and showing both the unit infection rates and the endemic prevalence of the microorganism.

The use of antiseptic soap (chlorhexidine) for hadwash as a routine was maintained throughout the study. MRSAinfected or -colonized patients were not isolated in private bedrooms on account of the unit lay-out. Such patients were identified in their records and/or in a visible place on their beds (Figure 1), for reinforcement of hygiene measures and barrier precautions, such as handwash, use of procedure gloves, overall, surgical masks and the utilization of individual equipment (stethoscope and sphygmomanometer) or, otherwise, to have such equipment cleaned with $70 \%$ alcohol. A newssletter with explanations on how to prevent and control MRSA infections was handed out to all professionals working in the unit (Figure 2).

All anterior nare colonized patients and professionals were submitted to a decolonization treatment with topical mupirocin (nasal Bactroban, SmithKline Beecham) - half a tube in each nare twice a day for 5 days, as of May 1997.

\section{Post-Intervention Group}

November 1, 1997 through June 30, 1998. The microorganisms were kept under daily surveillance, isolated

Figure 1. Identification card for MRSA patients.

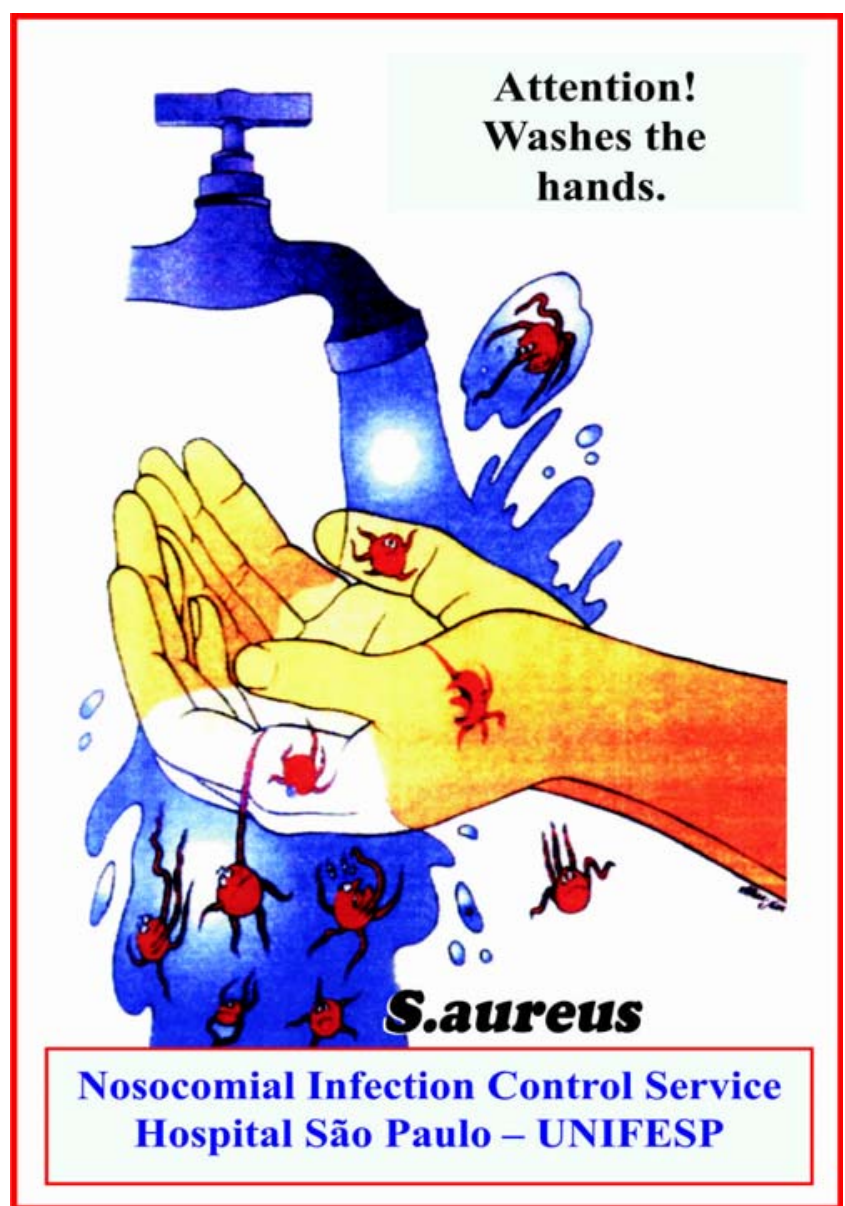


Figure 2. Bulletin with MRSA information.

\section{Multiresistant \\ Staphylococcus aureus \\ Do you know who that is?}

When oxacillin resistant the Staphylococcus aureus (MRSA) is also resistant to the other $\beta$-Inctoms (penicillin and by-products, cephalosporin, imipenem etc.). It is one of the most important corriers of hospital infections.

\section{Some of its characteristics}

1) High prevalence in our ICU ( $70 \%$ of the isolated S. oureus);

2) Rapid sprending within on institution or between hospitols;

3) Couses infection in ony environment;

4) High mortality rates.

Main Carriers - patients and healtheare professionals colonized or infected.

Transmission - direct contact, through hands of the healtheare workers. The bacterin can remain on the hands of the workers for minutes, hours or even longer.

How to control?

- Wash your hands with antiseptics or soap and water before and after handling ench patient; early identification of the colonized or infected patient;

- respect the isolation measures of contact established by CCIH;

- proceed to decolonization of professionals and patients when suggested by CCIH.

"Your participation is fundamental for control of hospital Infection"

Join us. CCIH

by the microbiology laboratory from all organic materials produced by ICU inpatients so as to diagnose MRSA infection, and to apply the NNISS methodology in the epidemiologic analysis of hospital infections. For three months within this period (January, February and March), we also assessed the inpatients for the prevalence of MRSA nasal carriers on a weekly basis, and only once the HCW. The isolated strains were tested for mupirocin susceptibility.

\section{Definitions}

A MRSA nasal carrier was defined as the patient or professional who had at least one positive anterior nare culture for the microorganism.

Infections were defined according to the criteria established by the Centers for Disease Control and Prevention (CDC) [18].

\section{Statistical Analysis}

For the analysis of the results, Student's " $t$ " tests were used for the average length of stay, and chi-square tests for non-parametrical data, comparing the control and postintervention periods.

\section{Results}

A total of 8,883 patients/day (pts/day) were assessed according to the NNISS methodology - of those, 1,660 (monthly mean $=332+14.08$ pts/day) in the control group, 4,072 (monthly mean $=339+51.45 \mathrm{pts} /$ day) in the intervention group and 3,151 (monthly mean $=393+35.08 \mathrm{pts} /$ day) in the post-intervention group.

The ratios of invasive device use (NNISS) per patient were the same for all three periods: control 2.20, at the intervention 2.26, and post-intervention $2.16(\mathrm{p}=0.61)$.

The incidence coefficients of per site ICU hospital infections (monthly median per 1,000 pts/day) in the control, intervention and post-intervention periods were respectively: global infection rate: $45.7,56.5,46.9(\mathrm{p}=0.85)$; bloodstream infection: $19.2,20.7,6.5$ per $1,000 \mathrm{cvc} /$ day $(\mathrm{p}<0.001)$; pneumonia: $25.5,28.6,14.2$ per 1,000 ventilator/day $(\mathrm{p}=0.006)$ and urinary tract infection: 10.6, 17.9, 14.0 per 1,000 urinary catheter/day ( $\mathrm{p}=0.36)$.

The methicillin-suseptible Staphylococcus aureus hospital infections remained virtually constant as we compared the control period, 3.01 infections per 1,000 pts/ day, with the post-intervention period, 0.9 infections per 1,000 pts/day ( $\mathrm{p}=0.09)$. However, increased Gram-negative bacilli (P. aeruginosa, A.baumannii, K. pneumoniae and Enterobacter cloacae) hospital infections: 21.1 infections/ $1,000 \mathrm{pts} /$ day (control) and 33.0 infections/1,000 pts/day (postintervention) $(\mathrm{p}=0.02)$.

The MRSA global rate of infection was 10.2, 5.1 and 2.5/ $1,000 \mathrm{pts} /$ day $(\mathrm{p}<0.001)$ and MRSA bloodstream infections were 3.6, 0.9 and 1.8/1,000 cvc/day ( $\mathrm{p}=0.281$ ) (Figure 3).

MRSA nasal colonization in ICU patients was 30.9 in the intervention period and 22.0 in the post-intervention period $(\mathrm{p}=0.06)$ (Table 1$)$.

Among the MRSA nasal carriers in the intervention group, $58.0 \%$ were positive at admittance to the ICU, $22.0 \%$ became positive in the first week and $20.0 \%$ from the second week on. In the post-intervention group, those rates were $46.1 \%, 26.9 \%$ and $26.9 \%$, respectively.

In regard to the nasal carrier vs. infection ratio in the intervention group, we found $76.2 \%$ of patients who developed MRSA hospital infection to be nasal carriers, whereas 23.8\% of the MRSA-infected patients carried no such microorganism in their nares. In the post-intervention group, $100 \%$ of the 
Figure 3. MRSA global and bloodstream infection rate in the control, intervention and post-intervention groups - ICU patients - São Paulo Hospital - UNIFESP - JUN/96 to JUN/ 98.

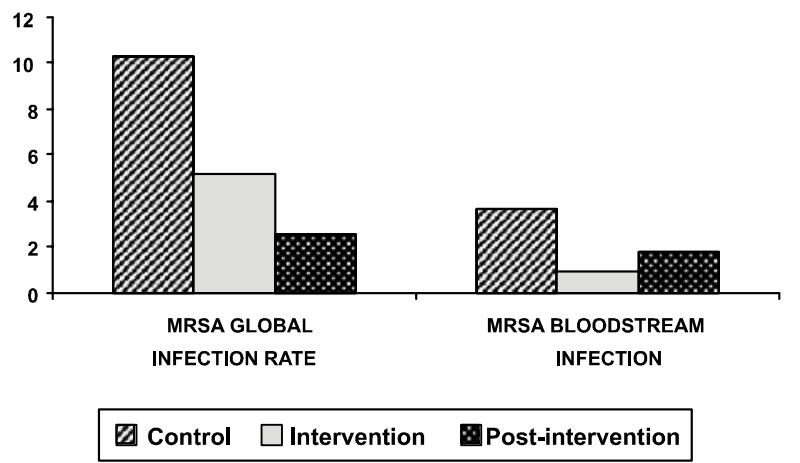

Table 2. ICU patients who developed MRSA infection in relation to the previous nasal carrier condition in the intervention and post-intervention groups - São Paulo Hospital - UNIFESP - NOV/96 to JUN/98

\begin{tabular}{|c|c|c|c|}
\hline & \multicolumn{3}{|c|}{ MRSA Infection } \\
\hline & $\begin{array}{c}\text { MRSAnasal } \\
\text { carriers } \\
\mathbf{N}^{0}(\%)\end{array}$ & $\begin{array}{c}\text { Non-carriers } \\
\text { No }^{\circ}(\%)\end{array}$ & $\begin{array}{c}\text { Total } \\
\mathbf{N}^{\circ}(\%)\end{array}$ \\
\hline Intervention group & 16 (76.19) & $5(23.80)$ & $21(100)$ \\
\hline $\begin{array}{l}\text { Post-intervention } \\
\text { group }\end{array}$ & $2(100.00)$ & $0(0.00)$ & $2(100)$ \\
\hline
\end{tabular}

Table 1. ICU patients evaluated with nare cultures for meticillin-resistant (MRSA) and methicillin-susceptible (MSSA) Staphylococcus aureus in the intervention and postintervention periods - São Paulo Hospital - UNIFESP - NOV/ 96 to JUN/98

\begin{tabular}{lcccc}
\hline Patients & $\mathbf{N}^{\mathbf{0}}$ & $\begin{array}{c}\text { MRSA } \\
\text { carriers } \\
\mathbf{N}^{\mathbf{0}} \mathbf{( \% )}\end{array}$ & $\begin{array}{c}\text { MSSA } \\
\text { carriers } \\
\mathbf{N}^{\mathbf{0}} \mathbf{( \% )}\end{array}$ & $\begin{array}{c}\text { Non- } \\
\text { carriers } \\
\mathbf{N}^{\mathbf{0}} \mathbf{( \% )}\end{array}$ \\
\hline Intervention & 333 & $103(30.9)$ & $48(14.4)$ & $182(54.7)$ \\
Post- intervention & 118 & $26(22.0)$ & $25(21.2)$ & $67(56.8)$
\end{tabular}

$\mathrm{P}=0.20$ (comparison among MRSA carriers in the intervention and post-intervention periods).

MRSA-infected patients were nare-colonized (Table 2). MRSA-nare colonized patients were at a $7.1(\mathrm{CI}-95 \%=2.7-$ 18.9) higher risk of developing infection caused by the microorganism than those not colonized in their nares.

The average time between colonization and development of infection was 8.5 ( 1 to 31 ) days and the median was 5.5 days.

Analysis of HCW for MRSA nasal colonization showed a $12.1 \%$ prevalence in the intervention period (Table 3 ), with predominance of nursing professionals (nurses and nurses' aids) over the others. In the post-intervention period, of the 108 HCWs assessed, 4.6\% were MRSA positive $(\mathrm{p}=0.037)$. No physicians, nurses or respiratory therapists were positive (Table 3).

Table 3. Healthcare workers MRSA nasal colonized in the intervention and post-intervention periods - São Paulo Hospital UNIFESP - MAR/97 to JUN/98

\begin{tabular}{lccccc}
\hline & \multicolumn{2}{c}{ Intervention period } & & Post-intervention period \\
\cline { 2 - 4 } \cline { 5 - 6 } & $\begin{array}{c}\text { Assessed } \\
\text { professionals }\end{array}$ & $\begin{array}{c}\text { MRSA } \\
\text { nasal carriers } \\
\mathbf{N}(\mathbf{\%})\end{array}$ & & $\begin{array}{c}\text { Assessed } \\
\text { professionals }\end{array}$ & $\begin{array}{c}\text { MRSA } \\
\text { nasal carriers } \\
\mathbf{N}(\%)\end{array}$ \\
\hline Nurse aides and Nurse technicians & 66 & $11(16.66)$ & & 70 & $5(7.14)$ \\
Physicians & 37 & $1(2.70)$ & & 14 & $0(0.00)$ \\
Registered Nurses & 36 & $6(16.66)$ & & 18 & $0(0.00)$ \\
Respiratory Therapists & 3 & $0(0.00)$ & & 2 & $0(0.00)$ \\
Administration/ Hygiene & 6 & $0(0.0)$ & & 4 & $0(0.0)$ \\
Total & 148 & $18(12.16)$ & & 108 & $5(4.62)$ \\
\hline
\end{tabular}

The efficacy of topical mupirocin treatment to eliminate nasal carriers in patients was 93.7\% (30/32), and 88.2\% (15/17) in HCWs.

The patients' tested strains showed 1.9\% (5/259) mupirocim resistance in the intervention group and $1.28 \%(1 / 78)$ in the post-intervention group ( $\mathrm{p}=0.709)$. In either period no mupirocin resistance in the isolated samples of HCW were founded.

The patients' ICU length of stay, in the three periods was $11.3,15.1$ and 12.7 days, respectively $(p=0,76)$.
The mortality rate (\%) in the three periods was respectively, 26.6, 27.2 and $21.0(\mathrm{p}<0.001)$.

Among the Staphylococcus aureus strains isolated from several sites in the three periods, respectively $64.5 \%, 84.6 \%$ and $71.1 \%$ of oxacillin resistance $(\mathrm{p}=0.31$ ) was found.

\section{Discussion}

Although we are aware that the acquisition of MRSA is an important event in the hospitalized patient, mainly in ICUs, little is known about the efficacy of control measures when 
the microorganism is endemic, as it occurs in most of the large Brazilian university hospitals.

The ICU studied is a service which attends to both clinical and surgical patients either from the emergency room or from other areas of the hospital. It poses high prevalence of MRSA and it is likely to spread the microorganism to other areas through the exchange of patients.

The adoption of MRSA control measures is highly discussed in the literature and their efficacy, and even the worthness of implementing them, is controversial $[4,6,9]$. The different needs to control the microorganism experienced by the institutions, associated with recent changes in its epidemiology, such as increased community cases [1] and the importance of environmental surface contamination, render the problem even more complex [19].

Control strategies should be developed by each institution based on factors such as: whether it is an endemic situation, an outbreak, the population of patients, the type of unit and the resources available for infection control. In low prevalence or non-endemic MRSA institutions, strict application of standard measures can effectively curb transmission [7]. The control program shown in the study, which included early identification of infected and colonized patients, guidance measures, modified contact isolation and treatment of nasal carriers with intranasal mupirocin ointment, was effective in reducing the incidence of bloodstream infections, pneumonia and hospital infections caused by MRSA in our ICU patients. The intervention made no impact on the length of stay in the unit, but it reduced the overall mortality rate significantly. Some studies show the efficacy of such measures in an endemic situation $[4,5,7,8]$, and outbreaks that were controlled with the implementation of such measures are also reported in the literature.

The isolation of MRSA-colonized or infected patients is a recommended measure, but difficult to be applied in our reality due to structural problems in the hospitals. Alternative isolation measures proved to be equally effective when applied to specific units [20,21].

MRSA nasal colonization in patients admitted to the ICU was high in the intervention period showing slight decline in the post-intervention period. Most of such patients were already colonized at admittance to the unit. When we evaluated the MRSA nare-positive patients alone, in the intervention period, $31.4 \%$ of such patients were found to have been inpatients for less than 48 hours, which corrobrates the relevance of assessing the MRSA carrier at admittance to a unit where control measures are being implemented, considering the growing prevalence that microorganism in the community [22]. Furthermore, in the intervention and postintervention groups respectively, $22.0 \%$ and $26.9 \%$ of patients were found to have become positive in the first week of admittance to the ICU, which shows the high risk of nasal MRSA acquisition in an endemic situation. MRSA nasal carriers rates rang from $5.6 \%$ to $12.9 \%$ in ICU patients [5,10,23], such rates being lower than the ones found in our study. As yet, little is known about the MRSA nasal carrier epidemiology in ICU patients or its subsequent clinical consequences, since few in-depth investigations have been made.

Murder et al. [24] evaluated the development of staphylococcal infection in methicillin-resistant (MRSA) and methicillin-susceptible (MSSA) S. aureus nasal carriers and found that $25 \%$ of patients who developed infection were MRSA carriers, whereas among MSSA carriers or non-carriers, as few as $4 \%$ and $4.5 \%$ respectively developed infections (73\% of all MRSA infections occurred in nasal carriers of the microorganism). They also concluded that the management of carriers may be used as a control measure.

We found an association between the MRSA nasal carrier and further development of infection caused by the microorganism in several sites. Of the patients who developed proven MRSA infection, 76.1\% carried the microorganism in their nares, accounting for a 7 times higher risk for that colonized patients. MRSA colonization and the risk of infection in critically ill patients has been described [24].

Pujol et al., in an ICU study, concluded that $S$. aureus nasal carriers are at a high risk for the development of bacteremia by the microorganism and suggest that treatment of those patients may be effective in controlling the agent [10].

Elimination of nasal carriage with topical mupirocin proved to be effective in our study, in agreement with other reports $[5,8,25]$. Mupirocin resistance was not significant in our study, probably due to its brief use.

The high prevalence of MRSA nare colonized HCW is an event not found by other authors in an endemic or epidemic situation, not even in risk units $[5,25,26]$. Of course in critical units, direct and long term contact between professionals and patients predisposes to increased colonization when high endemic rates are found. In the post-intervention period a nasal carriage decrease of approximately $60 \%$ was noticed among the professionals $(p=0.037)$ probably due to the decreased incidence of MRSA infection which could be a source of environmental contamination since those professionals had been exposed to the patients while attending them.

MRSA infections declined following implementation of the procedures. However, reduction of nasal colonization among patients, hasn't been observed even when only the ones who had become positive in the ICU were assessed.

As the number of MRSA nasal carriers decreased among the professionals, can we consider that they were not important as a reservoir and also in the transmission of the microorganism?

In order to compare the length of stay and the mortality rate among the patients hospitalized in the control and postintervention periods, we used the device use ratio as an indicator of the patients' nosological conditions.

Since those periods were equivalent, it may be said that no important changes occurred in the inpatients severity condition during our study.

Despite no changes to the length of hospital stay, a decrease in the overall mortality rate in the period subsequent 
to the procedures implementation was found, probably as a result of reduced pneumonia, bloodstream and MRSA infections, that are known to cause high mortality.

In conclusion, control measures should be introduced in risk areas of MRSA endemic hospitals, even if the procedures cannot be carried out throughout the institution. However, further studies should be conducted for the relevance of each procedure to be evaluated distinctly.

\section{Acknowledgement}

The authors thank Dr. José Luis Amaral and the team of doctors and nurses in ICU. Dr. Hélio Sader, Ms Odimara P Santos and Ms Iraci Tosim from the Special Laboratory of Microbiology (LEMC).

\section{References}

1. Vos M.C., Verbrugh H.A. MRSA: We can overcome, but who will lead the battle? Infect Control Hosp Epidemiol 2005;26:11720.

2. Hiramatsu K., Aritaka N., Hanaki H., et al. Dissemination in Japanese hospitals of strains of Staphylococcus aureus heterogeneously resitant to vancomycin. Lancet 1997;350:1670-3.

3. Moreira M., Medeiros E.A.S., Pignatiari A.C.C., et al. Efeito da infecção hospitalar da corrente sangüínea por Staphylococcus aureus resistente a oxacilina sobre a letalidade e o tempo de hospitalização. Rev Ass Med Brasil 1998;44:1-7.

4. Adeyemi-Doro F.A., Scheel O., Lyon D.J., Cheng A.F. Living with methicillin-resistant Staphylococcus aureus: a 7-year experience with endemic MRSA in a university hospital. Infect Control Hosp Epidemiol 1997;18:765-7.

5. Blumberg L.H., Klugman K.P. Control of methicillin-resistant Staphylococcus aureus bacteremia in high-risk areas. Eur J Clin Microbiol Infect Dis 1994;13:82-5.

6. Mylotte J.M. Control of methycillin-resistant Staphylococcus aureus: the ambivalence persists. Infect Control Hosp Epidemiol 1994; $15: 73-7$.

7. Muto C.A., Jernigan J.A., Ostronsky B.E., et al. Shea guideline for preventing nosocomial trasmision of multidrug-resistant strains of Staphylococcus aureus and Enterococcus. Infect Control Hosp Epidemiol 2003;24:362-86.

8. Barrett S.P., Mummery R.V., Chattopadhyay. Trying to control MRSA causes more problems than it solves. J Hosp Infect 1998;39:85-93.

9. Girou E., Pujade G., Legrand P., et al. Selective screening of carriers for control of meticillin-resistant Staphylococcus aureus (MRSA) in high-risk hospital areas with a high level of endemic MRSA. Clin Infect Dis 1998;27:543-50.

10. Pujol M., Peña C., Pallares R., et al. Nosocomial Staphylococcus aureus bacteremia among nasal carriers of methicillin-resistant and methicillin-susceptible strains. Am J Med 1996;100:509-16.
11. Talon D., Rouget C., Cailleaux V., et al. Nasal carriage of Staphylococcus aureus and cross-contamination in a surgical intensive care unit: efficacy of mupirocin ointment. J Hosp Infect 1995;30:39-49.

12. Hudson I.R.B. The efficacy of intranasal mupirocin in the prevention of staphylococcal infections: a review of recent experience. J Hosp Infect 1994;27:81-98.

13. Bertino J.S. Intranasal mupirocin for outbreak of methicillinresistant Staphylococcus aureus. Am J Health Syst Pharm 1997;54:2185-91.

14. Santos K.R.N., Fonseca L.S., Contijo Filho P.P. Emergence of High-Level Mupirucin Resistance in methicillin-resistant Staphylococcus aureus Isolated From Brazilian University Hospitals. Infect Control Hosp Epidemiol 1996;17:813-8.

15. Emori T.G., Culver D.H., Horan T.C., et al. National Nosocomial Infections Surveillance System (NNISS): description of surveillance methods. Am J Infect Control 1991;19:19-35.

16. National Comittee for Clinical Laboratory Standards (NCCLS) Methods for diluition antimicrobial susceptibility test for bacteria that grow aerobically, 4rd ed.: Approved standard, M7-A4. Villanova, PA.1997a.

17. National Comittee for Clinical Laboratory Standards (NCCLS) Performance standards for antimicrobial disk susceptibility tests, $6^{\text {th }}$ ed.:Approved standards, M2-A6. Villanova, PA.1997b.

18. Garner J.S., Jarvis W.R., Emori T.G., et al. CDC definitions for nosocomial infections, 1988. Am J Infect Control 1988;16:12840.

19. Boyce J.M., Potter-Bynoe G., Chenevert C., King T. Environmental contamination due to methicillin-resistant Staphylococcus aureus: possible infection control implications. Infect Control Hosp Epidemiol 1997;18:622-7.

20. Fazal B.A., Telzak E.E., Blum S., et al. Trends in the prevalence of methicillin-resistant Staphylococcus aureus associated with discontinuation of an isolation policy. Infect Control Hosp Epidemiol 1996;17:372-4.

21. Ribner B.S., Landry M.N., Gholson G.L. Strict versus modified isolation for prevention of nosocomial transmission of methicillin-resistant Staphylococcus aureus. Infect Control 1986; $7: 317-20$

22. Gould I.M. The clinical significance of methicillin-resistant Staphylococcus aureus. J Hosp Infection 2005;61:277-82.

23. Keene A., Vavagiakis P., Lee M-H., et al. Staphylococcus aureus colonization and the risk of infection critically ill patients. Infect Control Hosp Epidemiol 2005;26:622-8.

24. Muder R.R., Brennen C., Wagener M.M., et al. Methicillin-resistant staphylococcal colonization and infection in a long-term care facility. Ann Inter Med 1991;114:107-12.

25. Hartstein A.I., Denny M.A., Morthland V.H., et al. Control of methicillin-resistant Staphylococcus aureus in a Hospital and an intensive care unit. Infect Control Hosp Epidemiol 1995; $16: 405-11$.

26. Lingnau W., Allerberger F. Control of an outbreak of methicillinresistant Staphylococcus aureus (MRSA) by hygienic measures in a general intensive care unit. Infection 1994;22(suppl 2):135S9S. 